\title{
La creazione di valore: aspetti critici e problematiche di misurazione
}

\author{
Riccardo Mussari ${ }^{*}$,Andrea Melis ${ }^{* *}$, Patrizio Monfardini ${ }^{* * *}$
}

L'assunzione che la creazione di valore sia un obiettivo coerente con il perseguimento del fine ultimo dell'impresa, ovvero il soddisfacimento dei bisogni umani, non è nuova nella dottrina economico-aziendale, che da tempo si è posta il problema di sviluppare una teoria del valore ${ }^{1}$. In tale ottica, si pone il problema di come misurare il valore eventualmente creato tramite l'attività di produzione economica, ossia di definire il concetto di valore creato da parte di un'impresa e di individuare le tecniche e gli indicatori atti a stimare tale valore, che rappresenta una quantità economica congetturata.

Ampio è il dibattito esistente fra chi sostiene che l'impresa deve creare valore principalmente per i propri azionisti, e strumentalmente per i propri stakeholder strategici, e coloro i quali ritengono che sia il valore per gli stakeholder aziendali l'obiettivo ultimo che una sana e lungimirante gestione dovrebbe porsi ${ }^{2}$.

Anche qualora ci si focalizzi nel tentativo di stimare il valore creato in un'ottica "shareholder", si è sempre più consapevoli che il valore creato tramite la produzione economica sia difficilmente rappresentabile tramite la sola determinazione del reddito d'esercizio (ed il collegato capitale di funzionamento). Nonostante il processo di convergenza dei principi contabili in atto a livello internazionale, non esiste ancora una definizione univoca di reddito d'esercizio, essendo possibile misurarlo applicando diversi criteri di valutazione. Tali diversità possono determinare differenti risultati d'esercizio anche quando riferiti alla

* Università di Siena.

** Università di Cagliari.

**** Università di Cagliari.

${ }^{1}$ In merito, per un ulteriore approfondimento si rinvia a quanto scritto in A. Melis, Creazione di valore e meccanismi di corporate governance, Giuffré, Milano, 2002, ove ulteriori riferimenti dottrinali.

${ }^{2}$ Per un ulteriore riferimento a riguardo si rinvia, senza pretesa di esaustività, ai seguenti contributi: E. Freeman, G. Rusconi, M. Dorigatti (a cura di), Teoria degli stakeholder, FrancoAngeli, Milano, 2007; M. Jensen, Value maximization, stakeholder theory, and the corporate objective function, Journal of Business Ethics, Vol. 12, N. 2, 2002, pp. 235-256.

Management Control 2, 2012 - Special Issue 
medesima azienda ed agli stessi fatti amministrativi ${ }^{3}$. Il principio di unitarietà della gestione è parzialmente tradito nel momento in cui lo strumento di misurazione del reddito d'esercizio è costruito sul concetto di azienda come "finzione legale" ${ }^{4}$ ed aggregato di singoli cespiti in cui - con l'introduzione del criterio del fair value - il valore di mercato tende a sostituirsi al valore d'uso. Il concetto di unitarietà è parzialmente tradito anche nella sua prospettiva temporale poiché il postulato del going concern non è in grado di consentire una stima completa delle prospettive reddituali future, fermandosi a considerare i prevedibili esiti delle sole operazioni in corso.

Inoltre, la misurazione del valore creato tramite la produzione economica dovrebbe tener conto del rischio sotteso agli investimenti, rischio che grava sui flussi futuri di tipo reddituale e finanziario. A parte rare eccezioni (es. attività e della passibilità liquidabili entro o oltre l'esercizio) non vi è obbligo di comunicare all'esterno informazioni atte che il lettore del bilancio comprenda il grado di rischio che sottende certe valutazioni espresse in bilancio.

I problemi appena accennati in merito alla misurazione del valore creato presentano una valenza ancora più ampia di quella della semplice stima dal valore creato in quanto possono influenzare il processo stesso di creazione di valore, facendo sì che chi gestisce l'impresa sia disincentivato ad effettuare investimenti che, seppur utili all'effettiva creazione di valore, siano scarsamente apprezzati nella loro misurazione (su tutti gli investimenti in attività immateriali) $)^{5}$. Se tali difficoltà esistono nelle imprese private, in cui il lucro rappresenta un chiaro fine societario, esse divengono maggiori nelle aziende pubbliche.

Il valore pubblico è tema di ricerca affermato oltre che consueto argomento per il dibattito operativo ${ }^{6}$. Le amministrazioni pubbliche (AP), come tutte le altre aziende, sono chiamate a produrre e aggiungere valore tramite i processi di pro-

\footnotetext{
${ }^{3}$ Cfr. A. Melis, A. Mura, G. Roberto, S. Gaia, IAS/IFRS, US GAAP e principi contabili nazionali: un'analisi comparata empirica sui bilanci consolidati delle imprese italiane quotate anche alla borsa valori di New York, in L. Marchi, L. Potito (a cura di), L'impatto dell'adozione degli IAS/IFRS sui bilanci delle imprese italiane quotate, FrancoAngeli, Milano, 2012, ove ulteriori approfondimenti.

${ }^{4}$ Tale concetto è tipico della dottrina angloamericana e, di fatto, è ripreso dai principali standard-setters internazionali: FASB e IASB.

${ }^{5}$ A riguardo si vedano: R. Kaplan, Yesterday’s Accounting Undermines Production, Harvard Business Review, July-August, 1984, p. 101; R. Kaplan, D. Norton, The Balanced Scorecard. Translating Strategy into Action, Harvard Business School Press, Mass., 1996, 273.

${ }^{6}$ Le sintetiche riflessioni che seguono riprendono e, in parte, sviluppano considerazioni che abbiamo proposto in altri nostri scritti, fra i quali: Elio Borgonovi e Riccardo Mussari, Pubblico e privato: armonizzare gli opposti, Azienda Pubblica, XXIV, n. 2, 2011, pp. 103-121; Riccardo Mussari, Economia delle amministrazioni pubbliche, McGraw-Hill, Milano, 2011.
} 
duzione che pongono in essere. Muoversi nella prospettiva richiamata comporta più di un problema concettuale e di quantificazione (monetaria e non).

Infatti, è necessario distinguere fra valore pubblico proposto e valore pubblico riconosciuto ${ }^{7}$, definire l'ampiezza del tempo (breve, medio, lungo) cui riferire le due richiamate nozioni di valore e individuare i processi produttivi (solo alcuni o tutti) che in quel periodo si sono svolti.

Il valore proposto è definibile come la combinazione delle utilità incorporate nei beni e nei servizi realizzati in un dato periodo ed è quindi sintesi della quantità e della qualità degli output prodotti per essere ceduti. La misura del valore proposto dalle AP è convenzionalmente definita nei sistemi di contabilità pubblica e di contabilità nazionale come costi di produzione di competenza di un dato periodo. Tali costi possono essere riferiti ai processi di produzione di un servizio o congiuntamente di più servizi e derivano dalla contabilizzazione delle variazioni economiche relative ai fatti di gestione interna. Perciò essi sono influenzati dal grado di efficienza dei processi d'acquisto e d'impiego dei fattori produttivi. Anche se questa correlazione vale per ogni azienda, è opportuno evidenziare che ancora oggi le AP italiane possono avere difficoltà nel determinare il loro valore proposto essendo, in molti casi, ancora sprovviste del sistema di contabilità idoneo a rilevare in modo corretto i costi della produzione (contabilità economica analitica). In linea di principio, una volta definiti degli standard qualitativi minimi sia dei fattori produttivi acquisiti sia degli output realizzati, le AP dovrebbero tendere, a parità di volume di output, a ridurre il valore proposto. Accrescere l'efficienza comporta un contenimento, coeteris paribus, del valore proposto.

Il valore riconosciuto è indissolubilmente legato alla soddisfazione dei bisogni (domanda). Un bene o un servizio vale non solo poiché incorpora delle utilità, ma perché qualcuno lo richiede e utilizzandolo soddisfa, con diverso grado d'intensità, un proprio bisogno. Il valore proposto diventa riconosciuto se è accettato onde la dimensione di performance più direttamente coinvolta è l'efficacia, se partiamo dalla convinzione che gli obiettivi delle AP devono riflettere i bisogni pubblici ${ }^{8}$. Quando un'azienda vende i suoi prodotti tramite scambi di mercato, il consenso che ottiene è facilmente rilevabile: se un consumatore informato, potendo scegliere fra più proposte, predilige il prodotto offerto da una specifica azienda, egli esprime il suo consenso a quell'organismo socioeconomico con l'atto volontario dell'acquisto. In tali circostanze, la somma che il

${ }^{7}$ Sul tema, in generale, si rinvia a Giuseppe Catturi, L’azienda universale, Cedam, Padova, 2003.

${ }^{8}$ Sullo specifico argomento, rinviamo, fra gli altri, a Riccardo Mussari, La valutazione dei programmi nelle amministrazioni pubbliche, Giappichelli, Torino, 1999, p. 138 e segg. 
cliente di un'azienda è stato disposto a pagare per acquistare i suoi prodotti sono una misura attendibile del valore riconosciuto alla sua produzione. L'ipotesi sulla quale si fonda la nozione e la misura di valore riconosciuto è la libertà di scegliere da parte del consumatore; essa è generalmente valida perché lo scambio di mercato in situazioni di concorrenza è volontario e consegue a una scelta individuale e informata. Quando si opera sul mercato in condizioni di concorrenza, misurare in termini monetari valore proposto e valore riconosciuto permette un immediato riscontro della ragion d'essere di un'azienda. Se il valore proposto non è, almeno nel medio periodo, adeguatamente riconosciuto dall'ambiente cui è offerto, l'organismo socio-economico non è considerato in grado, attraverso la sua produzione, di soddisfare i bisogni delle persone alle quali si rivolge e, di conseguenza, la sua sopravvivenza è a rischio.

Per le AP le condizioni sopra richiamate (produzione per lo scambio di mercato in condizione di concorrenza e con consumatori adeguatamente informati) non ricorrono frequentemente e, pertanto, sarebbe errato trasferire sic et simpliciter il percorso logico sopra richiamato.

Quanto scritto porta a prendere atto che il ruolo delle AP non si limita alla produzione di servizi che devono soddisfare il consumatore finale quale contropartita del pagamento delle imposte ${ }^{9}$.

Mancano, per definizione, i legami quantitativo-monetario e temporale fra il pagamento delle imposte e l'utilizzo delle più importanti produzioni pubbliche (Giustizia, Sicurezza, Tutela della salute, Istruzione, Difesa, etc.) che identificano, in larga porzione, i cosiddetti "beni pubblici". Il singolo contribuente o gruppi di essi può (possono) ottenere nessun beneficio o benefici inferiori ai sacrifici (per la rinuncia al reddito conseguente alle imposte) nel rapporto economico-finanziario con lo Stato (e più in generale con le AP), perché tale rapporto non è volontario. D'altro canto, può bene avvenire il contrario: ovvero che si utilizzino servizi pubblici, anche abbondantemente, senza avere pagato le imposte (e senza averle evase). In sostanza, il beneficio che al singolo contribuente deriva come conseguenza del pagamento delle imposte non può e non deve essere valutato solo sul piano individuale. Si tratta di un'operazione non solo da inquadrare nella tipica logica egoistica del libero scambio di mercato ove ciascuna parte cerca di massimizzare il proprio tornaconto individuale, ma anche sul piano collettivo, cioè tenendo conto delle esternalità positive correlate a determinate produzioni pubbliche. Quando utilizziamo l'espressione "sul piano collettivo" non vogliamo stabilire alcun contrasto fra singolo utente e comunità cui lo stesso appartiene. Rimarcare che il singolo utente dei servizi

${ }^{9}$ Faremo qui riferimento alle sole imposte come principale tipologia di tributi destinata a finanziare la produzione di servizi a utilità collettiva. 
pubblici è anche parte irrinunciabile di una comunità che esprime bisogni e necessità perché soggetto collettivo serve a ricordare che:

a) il singolo trae beneficio dai servizi pubblici anche quando non li utilizza direttamente proprio in quanto componente di una comunità;

b) i bisogni di una comunità non possono, in alcun caso, essere rappresentati come la somma dei bisogni degli individui singoli che la compongono, ma sistema di singoli legati da relazioni storiche, culturali, sociali, psicologiche, spazio-temporali, ambientali, giuridiche, economiche (dimensione plurale) ${ }^{10}$. Avere già conseguito un titolo di studio o non avere figli in età scolare non esime dal contribuire, attraverso il pagamento delle imposte, al finanziamento della produzione del servizio istruzione. Ciò non solo e perché è obbligatorio pagare le imposte, ma anche perché vivere in una comunità di persone istruite è una condizione migliore per tutti i suoi membri rispetto a un'ipotetica situazione alternativa in cui studia solo chi ne ha voglia o chi se lo può permettere per condizioni di censo. Si noti che la "condizione migliore" è effetto delle esternalità positive conseguenti alle produzioni delle AP e si qualifica, a ben ragionare, come presupposto indispensabile per facilitare il riconoscimento di valore privato proposto da aziende con e senza scopo di lucro $^{11}$. Per avere immediato riscontro di quanto affermato, si pensi alle motivazioni che, spesso e con ragione, sono addotte per spiegare il parziale sviluppo economico del nostro Mezzogiorno (mancanza di infrastrutture, controllo del territorio da parte della malavita, particolare lentezza della "macchina amministrativa e giudiziaria”, etc.). È per le ragioni richiamate che alcune categorie di prestazioni rese dalle AP e finanziate con le imposte rappresentano veri e propri diritti (istruzione, salute e ricorso alla giustizia) sanciti dalla Costituzione e da altre leggi dello Stato o sono correlati all'imposizione di doveri (usufruire di alcuni servizi pubblici è obbligatorio: ad esempio l'istruzione o le vaccinazioni, denunce obbligatorie di reati) o di pene (detenzione in carcere, sanzioni pecuniarie e amministrative, etc.).

Per giungere, almeno sul piano concettuale, a dare un significato al valore pubblico riconosciuto, bisogna quindi andare oltre, molto oltre, il rapporto fra AP e singolo utente nel quale si sostanzia la dimensione singolare del valore pubblico riconosciuto.

${ }^{10}$ Per questa ragione le comunità, sin dai tempi antichissimi, si valgono di meccanismi di rappresentanza per designare chi deve rappresentarne gli interessi e trovare un corretto equilibrio fra sacrifici richiesti (prelievo fiscale) e benefici ricevuti (produzioni pubbliche).

${ }^{11}$ Pur se segue un percorso teorico e logico diverso, sull'argomento si rinvia a: John J. Kirlin, What Government Must Do Well: Creating Value for Society, Journal of Public Administration Research and Theory, Oxford University Press, Vol. 6, No. 1, 1996, pp. 161-185. 
Chiariamo bene. Non si vuole qui sostenere che le AP possano trascurare di soddisfare il consumatore finale nel singolo atto di erogazione di una prestazione; si vuole argomentare che ciò non esaurisce il significato di valore pubblico riconosciuto e che, a tal fine, occorre sforzarsi per dare un significato teorico ma anche operativo alla dimensione plurale del valore pubblico riconosciuto.

La dimensione plurale, comunitaria o sociale, del valore pubblico riconosciuto va ricondotta alla capacità delle AP di dare risposta ai bisogni collettivi della comunità di riferimento ${ }^{12}$. Le AP, infatti, sono chiamate a risolvere problemi (ridurre la disoccupazione, combattere la criminalità organizzata, preservare l'ambiente, tutelare il patrimonio artistico, sostenere lo sviluppo economico, rafforzare la coesione sociale, garantire la giustizia, ecc.) che interessano la comunità nel suo insieme (obiettivi sociali intesi come obiettivi della e per la società). Un'accettabile approssimazione della misura della dimensione plurale del valore pubblico riconosciuto è data dalla stima (in sede di programmazione) o dalla rilevazione (in sede di consuntivo) degli effetti sulla soluzione del problema oggetto delle politiche pubbliche, in altre parole il loro impatto.

Se questo punto di partenza è condivisibile, conseguono una serie di significative conseguenze sulle quali la migliore ricerca s'interroga:

a) il periodo di tempo di riferimento per la stima del valore pubblico riconosciuto non può essere breve perché gli effetti delle politiche e dei programmi pubblici si manifestano solo nel medio e lungo andare;

b) l'impatto delle politiche pubbliche, ovvero la dimensione plurale del valore pubblico riconosciuto, spesso non ha una misura monetaria onde il confronto con il valore proposto non è immediato;

c) le politiche pubbliche sono scelte da governi il cui agire riflette il sistema dei valori nei quali specifiche maggioranze politiche si riconoscono e che caratterizzano e distinguono l'azione di governo delle AP. Ciò significa che valore pubblico e valori pubblici sono molto correlati in una relazione non necessariamente duratura ${ }^{13}$.

I quattro articoli che seguono in questo numero speciale sono stati selezionati tra gli oltre trenta presentati al Workshop Aidea Giovani tenutosi a Cagliari il 18 e 19 novembre 2011, avente come titolo "Creazione di valore: aspetti critici e problematiche di valutazione".

${ }^{12}$ Cfr. Barry Bozeman,Public Value and Public Interest: Counterbalancing Economic Individualism, Georgetown University Press, Washington DC, 2007.

${ }^{13}$ Cfr. R.A.W. Rhodes and John Wanna, Bringing the Politics Back In: Public Value in Westminster Parliamentary Government, Public Administration, Vol. 87, No. 2, 2009, pp. 161-183. 
Il workshop ha visto la partecipazione di numerosi giovani studiosi delle tematiche aziendali provenienti da tutta Italia. Tra tutti paper presentati, quattro lavori sono stati selezionati attraverso un processo di doppio referaggio cieco per essere pubblicati in questo numero speciale. Tali lavori offrono interessanti prospettive sul tema in oggetto, sia relativamente all'ambito pubblico largamente inteso, sia al contesto delle aziende private con un particolare approfondimento su quelle bancarie.

Il lavoro di Nisio, De Carolis e Losurdo dell'Università di Bari dal titolo "Il ciclo della performance negli Enti Locali: un'analisi empirica sull'adozione del Piano della Performance" affronta il tema della diffusione di uno dei più recenti strumenti di programmazione della performance per gli Enti Locali italiani previsto dalla normativa nazionale. Il Piano della Performance è il documento fondamentale per la costruzione del Ciclo della Performance previsto del decreto legislativo n. 150 del 2009. Si tratta del più recente tentativo del legislatore di introdurre, all'interno degli Enti Locali e, più in generale, delle pubbliche amministrazioni italiane, le logiche e gli strumenti del performance management. Gli Autori hanno pertanto analizzato la disponibilità di tale documento, sui siti web istituzionali degli Enti Locali italiani di dimensioni comprese tra 20mila e 250mila abitanti, sfruttando il fatto che la norma ne impone agli Enti la pubblicazione online. Il successivo approfondimento ha riguardato la conformità del Piano con le previsioni della citata norma e con le direttive della Civit. Il quadro che emerge dall'analisi empirica è di una scarsa compliance con la norma; pochi Enti hanno adottato il Piano così come richiesto dalla legge, sia in termini di contenuto che di pubblicazione online. La lettura che gli Autori forniscono dei risultati è che benché sia trascorso un tempo limitato dalla statuizione dell'obbligo, l'evidenza empirica mostra tutte le difficoltà ed, in alcuni casi le resistenze, che gli Enti oppongono al dettato normativo, confermando quanto ancora sia necessario investire in termini di risorse, formazione e strumenti perché la cultura del risultato e della misurazione delle performance possano attecchire negli Enti Locali italiani. Il lavoro di Serra, Mulas e Mura dell'Università di Cagliari dal titolo "Contribuzione pubblica e misurazione del Valore Aggiunto nelle imprese italiane: un'analisi empirica" affronta un tema di particolare interesse sia per le aziende pubbliche che per quelle private, soprattutto in una fase di crisi come quella attuale. Gli Autori mirano a comprendere gli effetti sul valore aggiunto aziendale derivanti dall'ottenimento di contributi pubblici. La rilevanza del tema è evidente: spendere risorse pubbliche per "aiutare" le imprese, genera gli attesi risultati positivi o si tratta di un utilizzo poco efficace quando non dannoso? Lo studio è stato condotto in un ambito temporale di 8 anni prendendo a riferimento oltre 220 aziende manifatturiere divise in due gruppi: le destinatarie di contributi pubbli- 
ci e le aziende non destinatarie di trasferimenti pubblici, incluse nel gruppo di controllo. I risultati dei test statistici sono coerenti con parte della letteratura sul tema e dimostrano che la contribuzione pubblica non incrementa il valore aggiunto aziendale, conducendo talvolta a riduzioni di efficienza. Ne consegue che, secondo gli Autori, le politiche pubbliche di sostegno alle imprese dovrebbero essere attentamente riconsiderate, per evitare gli effetti perversi sulla performance aziendale che i dati dell'analisi mostrano.

L'ambito sanitario costituisce il settore di indagine per il lavoro di Boscolo, Canobbio e Ciani dell'Università Bocconi di Milano dal titolo "Hospital-based HTA: quale valore per la valutazione delle tecnologie nelle Aziende Sanitarie italiane?”. L'articolo approfondisce il tema della valutazione delle tecnologie sanitarie in Italia attraverso la somministrazione di un questionario semistrutturato cui hanno risposto 61 strutture sanitarie. La valutazione delle tecnologie sanitarie, o HTA (Health Technology Assessment) è uno strumento di policy, la cui diffusione nel nostro paese è abbastanza recente e che mira a consentire alle aziende sanitarie la valutazione delle tecnologie più adatte e convenienti nei diversi ambiti di operatività. L'analisi empirica mostra una diffusione limitata ma, comunque, crescente di tale pratiche, con alcuni enti che hanno non solo già provveduto alla costituzione di una commissione di valutazione delle tecnologie esistenti, ma anche a collegare l'HTA alle procedure di acquisto dei nuovi strumenti.

Infine il lavoro di Ginesti e Sannino della Seconda Università di Napoli e di Tartaglia Polcini dell’Università Salerno dal titolo “Regole contabili e performance aziendale: impairment e disclosure degli strumenti rappresentativi di capitale AFS nei bilanci delle banche quotate italiane" affronta il tema della rappresentazione contabile delle poste dell'attivo cosiddette disponibili per la vendita. Il tema, particolarmente rilevante nell'attuale fase di crisi, riguarda i comportamenti contabili delle aziende bancarie quotate con riferimento alle perdite di valore delle loro attività disponibili alla vendita. Tali perdite possono, alternativamente, essere imputate ad una riserva di patrimonio (oppure alla voce other comprehensive income) oppure diminuire il cosiddetto net income.

L'oggetto di studio è l'analisi delle soglie di riduzione di valore che le banche quotate hanno ritenuto rilevanti per procedere, i conseguenti comportamenti contabili e la dislosure degli stessi. I risultati cui il paper perviene sono interessanti; evidenziano infatti i comportamenti, talvolta opportunistici, che gli attori aziendali mettono in campo durante la crisi ed il ruolo importante delle autorità di vigilanza per mitigare la variabilità delle scelte contabili.

I quattro paper appena descritti offrono al lettore interessanti spunti di riflessione sui temi della creazione del valore e sulla sua misurazione consentendo ulteriori studi e ricerche sul tema. 